\title{
Exactly solvable model of three interacting particles in an external magnetic field
}

\author{
E. P. Nakhmedov ${ }^{1,2,6}$, K. Morawetz ${ }^{1}$, M. Ameduri ${ }^{3,4}$, A. Yurtsever ${ }^{5}$, C. Radehaus ${ }^{6}$ \\ ${ }^{1}$ Max Planck Institute for the Physics of Complex Systems, Nöthnitzer Str. 38, 01187 Dresden, Germany \\ ${ }^{2}$ Azerbaijan Academy of Sciences, Institute of Physics, H. Cavid 33, 370143 Baku, Azerbaijan \\ ${ }^{3}$ Laboratory of Elementary Particle Physics, Cornell University, Ithaca, NY 14853, USA \\ ${ }^{4}$ Weill Cornell Medical College in Qatar, Qatar Foundation, Doha, Qatar \\ ${ }^{5}$ Dept. of Mathematics, Fatih University, Büyükçekmece, Istanbul, Turkey \\ ${ }^{6}$ Technische Universität Chemnitz, D-09107 Chemnitz
}

\begin{abstract}
The quantum mechanical problem of three identical particles, moving in a plane and interacting pairwise via a spring potential, is solved exactly in the presence of a magnetic field. Calculations of the pair-correlation function, mean distance and the cluster area show a quantization of these parameters. Especially the pair-correlation function exhibits a certain number of maxima given by a quantum number. We obtain Jastrow pre-factors which lead to an exchange correlation hole of liquid type, even in the presence of the attractive interaction between the identical electrons.
\end{abstract}

\section{INTRODUCTION}

The Laughlin wave function $[1,2]$ was introduced to describe the ground state of a two-dimensional (2D) longrange correlated electron gas in the fractional quantum Hall regime. The strong short-distance repulsion between any two particles is described by the Jastrow prefactor in the wave function. The same Jastrow prefactor appears also in the exact wave functions for $N$ particles interacting pairwise through a potential of the form $1 /\left(x_{i}-x_{j}\right)^{2}$, first obtained in one dimension by Calogero $[3,4]$, and later extended to two and higher dimensions [5-7]. These wave functions lead to the Wigner-Dyson distribution function [8] for $N$ particles.

A natural question therefore is whether the origin of this Jastrow prefactor is due to special features of the interactions mentioned above, or it is a generic property of models with short-range interactions.

The recent advances in the theory of disordered systems show that the distribution function of physical parameters of the mesoscopic system, such as the density of states (DOS) or the conductance, is represented by the Wigner-Dyson function in the weak localization regime (see, e.g., [9] for a review). Physically, this describes a repulsion between the energy levels. Averaging the partition function of a disordered system over the Gaussiandistributed one-particle impurity potentials results in an effective Hamiltonian with short-range interactions. This clearly shows that level repulsion is not a property of just few special interaction potentials.

In a recent paper [10], a new mechanism for the formation of three-particle clusters in Si-MOSFET structures and GaAs/AlGaAs heterojunctions has been studied. The exchange type interactions between $2 D$ band electrons in the inversion layer and charged impurities in the oxide of the MOSFET can lead to an effective three-particle attractive interaction. A qualitative analysis shows that the ground state of the three particles be- comes unstable with respect to arbitrary small attractive interactions between them. An attraction between three particles leads to the formation of bound states with negative energies [10]. Although a weak attractive threeparticle interaction does not produce a transition to a liquid state of the electron gas as described in Laughlin's approach, the ground state with three-particle clustering could be energetically favored in the fractional quantum Hall regime. This is a consequence of the occurrence of an effective pairing [10], which further reduces the ground state energy.

It is interesting to study the internal structure of a particular cluster with three particles, in order to find out if its wave function contains a Jastrow prefactor. This would prevent the particles from approaching each other even in the attractive case. In this paper we study this question by modeling a generic three-particle attractive potential by a pairwise spring-like interaction. We show that this choice of potential allows for an exact solution even in the presence of a constant external magnetic field.

Exact results obtained for solvable models are important to understand general features of three-particle problems and to test approximate solutions, such as the ones obtained from the widely used Fadeev's equations [11]. Very few exact solutions of three-particle problems have been found, mostly for one-dimensional [12-14] or for three-dimensional [15-17] spinless particles. In this paper we present an exact solution for a model of particles with spin.

The topology of the three-particle cluster may be either of a string form, when two particles with opposite spin are placed in the same spatial point, or of a triangle form when all three particles lie far from each others. In the former case the number of the spin configurations is equal to two (doublet states) with total spin $S=1 / 2$. Apart from this doublet states, a triangular clustering of quartet states may be also realized. This would correspond to four different symmetric spin wave functions with a total 
spin $S=3 / 2$. In a strong magnetic field this quartet states are the only relevant ones.

\section{THE MODEL}

The Hamiltonian of the model is

$$
\begin{aligned}
\hat{H}= & -\frac{\hbar^{2}}{2 m^{*}} \sum_{\alpha=1}^{3}\left(\frac{\partial}{\partial \mathbf{r}_{\alpha}}-i \frac{e}{\hbar c} \mathbf{A}_{\alpha}\right)^{2} \\
& +\frac{\kappa}{2}\left[\left(\mathbf{r}_{1}-\mathbf{r}_{2}\right)^{2}+\left(\mathbf{r}_{1}-\mathbf{r}_{3}\right)^{2}+\left(\mathbf{r}_{2}-\mathbf{r}_{3}\right)^{2}\right]
\end{aligned}
$$

where $\mathbf{r}_{\alpha}(\alpha=1,2,3)$ are the two-dimensional $(2 D)$ position vectors of electrons with effective mass $m^{*}$. The spring strength is chosen as $\kappa=m^{*} \omega_{0}^{2} / 3$ where $\omega_{0}$ is the specific frequency of the electron's relative vibration. $\mathbf{A}_{\alpha}=\frac{B}{2}\{-y, x, 0\}$ is the symmetric-gauge vector potential at the position of the $\alpha$-th particle for a magnetic field $\mathbf{B}=\{0,0, B / 2\}$ perpendicular to the electronic inversion layer.

In order to diagonalize the Hamiltonian we introduce the new coordinates $\{\mathbf{r}, \xi, \eta\}$,

$$
\begin{aligned}
\mathbf{r} & =\frac{1}{\sqrt{3}}\left(\mathbf{r}_{1}+\mathbf{r}_{2}+\mathbf{r}_{3}\right), \\
\xi & =\frac{1}{\sqrt{2}}\left(\mathbf{r}_{1}-\mathbf{r}_{2}\right), \\
\eta & =\sqrt{\frac{2}{3}}\left(\frac{\mathbf{r}_{1}+\mathbf{r}_{2}}{2}-\mathbf{r}_{3}\right),
\end{aligned}
$$

where $\mathbf{r}$ is the center-of-mass coordinate, $\xi$ is the relative coordinate of the particles 1 and 2 and $\eta$ is the relative coordinate of the third particle with respect to the centerof-mass of the particles 1 and 2 . In the new coordinates, the Hamiltonian is decoupled,

$$
\hat{H}=\hat{H}_{\mathbf{r}}+\hat{H}_{\xi}+\hat{H}_{\eta},
$$

where

$$
\begin{aligned}
& \hat{H}_{\mathbf{r}}=-\frac{\hbar^{2}}{2 m^{*}} \frac{\partial^{2}}{\partial \mathbf{r}^{2}}+\frac{3 \hbar^{2}}{8 m^{*} l_{B}^{4}} \mathbf{r}^{2}+i \frac{\hbar^{2}}{2 m^{*} l_{B}^{2}}\left(\mathbf{r} \times \nabla_{\mathbf{r}}\right)_{z} \\
& \hat{H}_{\xi}=-\frac{\hbar^{2}}{2 m^{*}} \frac{\partial^{2}}{\partial \xi^{2}}+\left(\frac{\hbar^{2}}{8 m^{*} l_{B}^{4}}+\frac{3 \kappa}{2}\right) \xi^{2}+i \frac{\hbar^{2}}{2 m^{*} l_{B}^{2}}\left(\xi \times \nabla_{\xi}\right)_{z} \\
& \hat{H}_{\eta}=-\frac{\hbar^{2}}{2 m^{*}} \frac{\partial^{2}}{\partial \eta^{2}}+\left(\frac{\hbar^{2}}{8 m^{*} l_{B}^{4}}+\frac{3 \kappa}{2}\right) \eta^{2}+i \frac{\hbar^{2}}{2 m^{*} l_{B}^{2}}\left(\eta \times \nabla_{\eta}\right)_{z}
\end{aligned}
$$

The last terms in the Hamiltonians given by Eqs. (4)-(6) are proportional to the angular momentum and $l_{B}^{2}=\frac{\hbar c}{e B}$ is the magnetic length.

The wave function $\Psi$ of the initial Hamiltonian, Eq. (1), can be presented as the product of three wave functions $\psi$ which are eigenfunctions of Eqs. (4)-(6)

$$
\Psi\left(\mathbf{r}_{1}, \mathbf{r}_{2}, \mathbf{r}_{3}\right)=\psi(r) \psi(\xi) \psi(\eta)
$$

Schrödinger's equation $\hat{H} \psi=E \psi$ with the Hamiltonian given by one of the Eqs. (4)-(6) is easily solved in a polar coordinate system where, e.g., Eq. (6) is written as

$$
\begin{aligned}
\hat{H}_{\rho}= & -\frac{\hbar^{2}}{2 m^{*}}\left(\frac{\partial^{2}}{\partial \rho^{2}}+\frac{1}{\rho} \frac{\partial}{\partial \rho}+\frac{1}{\rho^{2}} \frac{\partial^{2}}{\partial \varphi^{2}}\right)-i \frac{\hbar^{2}}{2 m^{*} l_{B}^{2}} \frac{\partial}{\partial \varphi} \\
& +\left(\frac{\hbar^{2}}{8 m^{*} l_{B}^{4}}+\frac{3 \kappa}{2}\right) \rho^{2}
\end{aligned}
$$

and $\rho$ and $\varphi$ are the radial and angular variables in the polar coordinate systems.

The Hamiltonian (8) commutes with the angular momentum operator $\hat{L}_{z}=-i \hbar \frac{\partial}{\partial \varphi},\left[\hat{H}_{\rho}, \hat{L}_{z}\right]=0$. Therefore the wave function $\psi(\rho, \varphi)$ of the Hamiltonian $\hat{H}_{\rho}$ can be chosen to be of the form

$$
\psi_{m}(\rho, \varphi)=e^{i m \varphi} R(\rho)
$$

where $m$ is the angular momentum for a $2 D$ system and $m= \pm 1, \pm 2, \pm 3 \ldots$.

The asymptotic behavior of the radial wave function $R(\rho)$ can be separated as

$$
\bar{R}(z)=z^{\frac{|m|}{2}} e^{-\frac{1}{2} z} f(z)
$$

where $z=\frac{\rho^{2}}{2 l_{B}^{2}} \sqrt{1+\frac{4 \omega_{0}^{2}}{\omega_{B}^{2}}}$ is a dimensionless coordinate, and $\omega_{B}=\frac{e B}{m^{*} c}$ is the cyclotron frequency.

The function $f(z)$ satisfies the confluent hypergeometric equation

$$
f(z)=F\left\{-\left(\frac{\beta}{\sqrt{1+4 \omega_{0}^{2} / \omega_{B}^{2}}}-\frac{1}{2}(|m|+1)\right), 1+|m|, z\right\}
$$

where $\beta=\frac{E}{4 \omega_{B}}-\frac{m}{2}$ is the dimensionless energy spectrum. Convergence requires the first coefficient of the confluent hypergeometric function to be a negative integer. The function then reduces to the generalized Laguerre polynomials and gives the following energy spectrum

$$
E_{n_{i}, m_{i}}=\hbar \omega_{B} \sqrt{1+\frac{4 \omega_{0}^{2}}{\omega_{B}^{2}}}\left(n_{i}+\frac{\left|m_{i}\right|+1}{2}\right)+\frac{\hbar \omega_{B}}{2} m_{i}
$$

where $i=1,2,3$ denotes the number of non-interacting quasi-particles introduced by means of a normal coordinate transformation. The total energy of a threeparticle cluster is expressed as a sum of the energies of three non-interacting quasi-particles. Redefining the initial quantum numbers as $n_{0}=n_{1}+\frac{1}{2}\left(\left|m_{1}\right|+m_{1}\right)$, $n=2\left(n_{2}+n_{3}\right)+\left|m_{2}\right|+\left|m_{3}\right|$ and $m=m_{2}+m_{3}$, the total energy becomes dependent on three exact quantum numbers, 


$$
\begin{aligned}
E\left(n_{0}, n, m\right)= & \hbar \omega_{B}\left(n_{0}+\frac{1}{2}\right) \\
& +\frac{\hbar \omega_{B}}{2} \sqrt{1+\frac{4 \omega_{0}^{2}}{\omega_{B}^{2}}}(n+2)+\frac{\hbar \omega_{B}}{2} m .
\end{aligned}
$$

The case corresponding to three free particles in an external magnetic field is given by the two exact quantum numbers $n=\sum_{i=1}^{3}\left(n_{i}+\left|m_{i}\right| / 2\right)$ and $m=\sum_{i=1}^{3} m_{i}$. The normalized wave function of the $i$-th quasi-particle can be written in the form

$$
\begin{aligned}
& \psi_{n_{i}, m_{i}}(\rho, \varphi)=\frac{1}{\sqrt{\pi}}\left(\frac{\sqrt{1+\frac{4 \omega_{0}^{2}}{\omega_{B}^{2}}}}{2 l_{B}^{2}}\right)^{\frac{\left|m_{i}\right|+1}{2}}\left[\frac{n !}{\left(n_{i}+\left|m_{i}\right|\right) !}\right]^{1 / 2} \\
& \times e^{i m_{i} \varphi} \rho^{\left|m_{i}\right|} \mathrm{e}^{-\sqrt{1+\frac{4 \omega_{0}^{2}}{\omega_{B}^{2}}} \frac{\rho^{2}}{4 l_{B}^{2}}} L_{n_{i}}^{\left|m_{i}\right|}\left(\sqrt{1+\frac{4 \omega_{0}^{2}}{\omega_{B}^{2}}} \frac{\rho^{2}}{2 l_{B}^{2}}\right) \cdot
\end{aligned}
$$

In order to construct the total wave function for a three particles cluster, the spatial wave functions corresponding to Eqs. (4)-(6) must be multiplied by the appropriate spin wave functions and the final expression must be antisymmetric.

\section{WAVE FUNCTIONS AND CORRELATORS}

All spin wave functions of the quartet states $\mid S=$ $\left.\frac{3}{2}, s_{z}\right\rangle$, corresponding to the total spin number $S=3 / 2$ and its $z$-component $s_{z}$, are symmetric

$$
\begin{aligned}
& |3 / 2,3 / 2\rangle=\alpha_{1} \alpha_{2} \alpha_{3} \\
& |3 / 2,1 / 2\rangle=\frac{1}{\sqrt{3}}\left\{\alpha_{1} \alpha_{2} \beta_{3}+\alpha_{1} \beta_{2} \alpha_{3}+\beta_{1} \alpha_{2} \alpha_{3}\right\} \\
& |3 / 2,-1 / 2\rangle=\frac{1}{\sqrt{3}}\left\{\alpha_{1} \beta_{2} \beta_{3}+\beta_{1} \beta_{2} \alpha_{3}+\beta_{1} \alpha_{2} \beta_{3}\right\} \\
& |3 / 2,-3 / 2\rangle=\beta_{1} \beta_{2} \beta_{3}
\end{aligned}
$$

where $\alpha_{i}$ and $\beta_{i}$ are the spinors of the $i$-th electron corresponding to spin up and down, respectively. Therefore, the antisymmetric orbital part of the wave function reads

$$
\begin{aligned}
& \Psi^{Q}\left(\mathbf{r}_{\mathbf{1}}, \mathbf{r}_{\mathbf{2}}, \mathbf{r}_{\mathbf{3}}\right)=C \Psi_{n_{1}, m_{1}}\left(r ; \omega_{0}=0\right) \\
& \times\left\{\Psi_{n_{2}, m_{2}}(\xi) \Psi_{n_{3}, m_{3}}(\eta)-\Psi_{n_{2}, m_{2}}(-\xi) \Psi_{n_{3}, m_{3}}(\eta)\right. \\
& +\Psi_{n_{2}, m_{2}}(\tilde{\xi}) \Psi_{n_{3}, m_{3}}(\tilde{\eta})-\Psi_{n_{2}, m_{2}}(-\tilde{\xi}) \Psi_{n_{3}, m_{3}}(\tilde{\eta}) \\
& \left.+\Psi_{n_{2}, m_{2}}(\tilde{\tilde{\xi}}) \Psi_{n_{3}, m_{3}}(\tilde{\tilde{\eta}})-\Psi_{n_{2}, m_{2}}(-\tilde{\tilde{\xi}}) \Psi_{n_{3}, m_{3}}(\tilde{\tilde{\eta}})\right\},
\end{aligned}
$$

where $C$ is a normalization constant and we have used the notation

$$
\begin{aligned}
& \tilde{\xi}=\frac{\mathbf{r}_{\mathbf{2}}-\mathbf{r}_{\mathbf{3}}}{\sqrt{2}}=\frac{\sqrt{3}}{2} \eta-\frac{\xi}{2}, \\
& \tilde{\eta}=\sqrt{\frac{2}{3}}\left(\frac{\mathbf{r}_{\mathbf{2}}+\mathbf{r}_{\mathbf{3}}}{2}-\mathbf{r}_{\mathbf{1}}\right)=-\frac{\eta}{2}-\frac{\sqrt{3}}{2} \xi,
\end{aligned}
$$

$$
\begin{aligned}
& \tilde{\tilde{\xi}}=\frac{\mathbf{r}_{\mathbf{3}}-\mathbf{r}_{\mathbf{1}}}{\sqrt{2}}=-\frac{\sqrt{3}}{2} \eta-\frac{\xi}{2}, \\
& \tilde{\tilde{\eta}}=\sqrt{\frac{2}{3}}\left(\frac{\mathbf{r}_{\mathbf{1}}+\mathbf{r}_{\mathbf{3}}}{2}-\mathbf{r}_{\mathbf{2}}\right)=-\frac{\eta}{2}+\frac{\sqrt{3}}{2} \xi .
\end{aligned}
$$

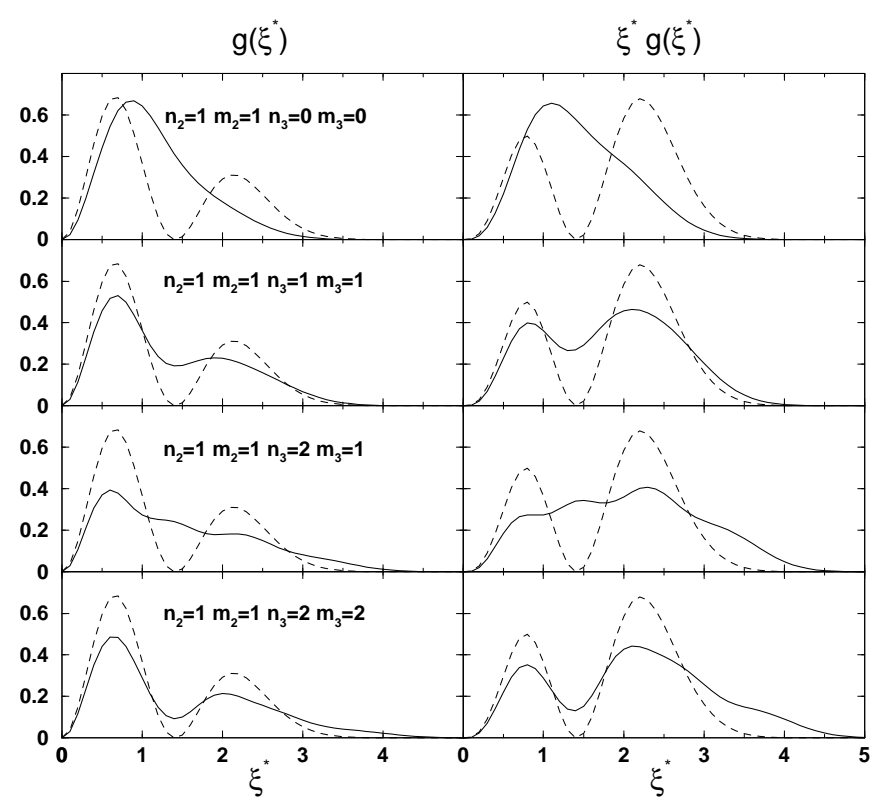

FIG. 1. The normalized pair correlation function $g\left(\xi^{*}\right)$ versus the dimensionless distance between two particles $\xi^{*}$ for $n_{2}=1$ and different values of the quantum numbers $n_{3}, m_{3}$. The dotted lines are the pair correlation function only from the diagonal elements of $|\Psi|^{2}$ according to (25).

Notice that the inversion of the coordinates in polar basis leads to $\rho \rightarrow \rho$ and $\varphi \rightarrow \varphi+\pi$. Under this transformation, the wave function acquires a factor $(-1)^{m}$, which defines its parity. It is easy to see that $\Psi^{A}\left(\mathbf{r}_{\mathbf{1}}, \mathbf{r}_{\mathbf{2}}, \mathbf{r}_{\mathbf{3}}\right)=0$ for $m_{2}=$ even integer. The privileged dependence of the wave function on $m_{2}$ is originated by the asymmetry in the new coordinates (2). For $m_{2}=$ odd integer, we get

$$
\begin{aligned}
\Psi^{Q}\left(\mathbf{r}_{\mathbf{1}}, \mathbf{r}_{\mathbf{2}}, \mathbf{r}_{\mathbf{3}}\right)= & C \Psi_{n_{1}, m_{1}}\left(\mathbf{r} ; \omega_{o}=0\right) \\
\times & \left\{\Psi_{n_{2}, m_{2}}(\xi) \Psi_{n_{3}, m_{3}}(\eta)\right. \\
& +\Psi_{n_{2}, m_{2}}(\tilde{\xi}) \Psi_{n_{3}, m_{3}}(\tilde{\eta}) \\
& \left.+\Psi_{n_{2}, m_{2}}(\tilde{\tilde{\xi}}) \Psi_{n_{3}, m_{3}}(\tilde{\tilde{\eta}})\right\} .
\end{aligned}
$$

In high magnetic fields the polarized spin structure is characterized by this quartet states since all states are aligned. The valuable feature of the spin-polarized quartet states is that they permit only odd values of $m_{2}$ and exclude even ones, which is the situation observed in the fractional quantum Hall experiments. The wave function $\Psi^{Q}$ given by Eq. (21) has the property that $\left.\Psi^{Q}\left(\mathbf{r}_{\mathbf{1}}, \mathbf{r}_{\mathbf{2}}, \mathbf{r}_{\mathbf{3}}\right)\right|_{\mathbf{r}_{\mathbf{i}} \rightarrow \mathbf{r}_{\mathbf{j}}}=0$ for arbitrary chosen $i \neq j=$ $1,2,3$. This corresponds to the behavior of the Jastrow prefactor $f\left(\mathbf{r}_{\mathbf{i}}-\mathbf{r}_{\mathbf{j}}\right)$ in Laughlin's ground state wave function $[1,2]$. 


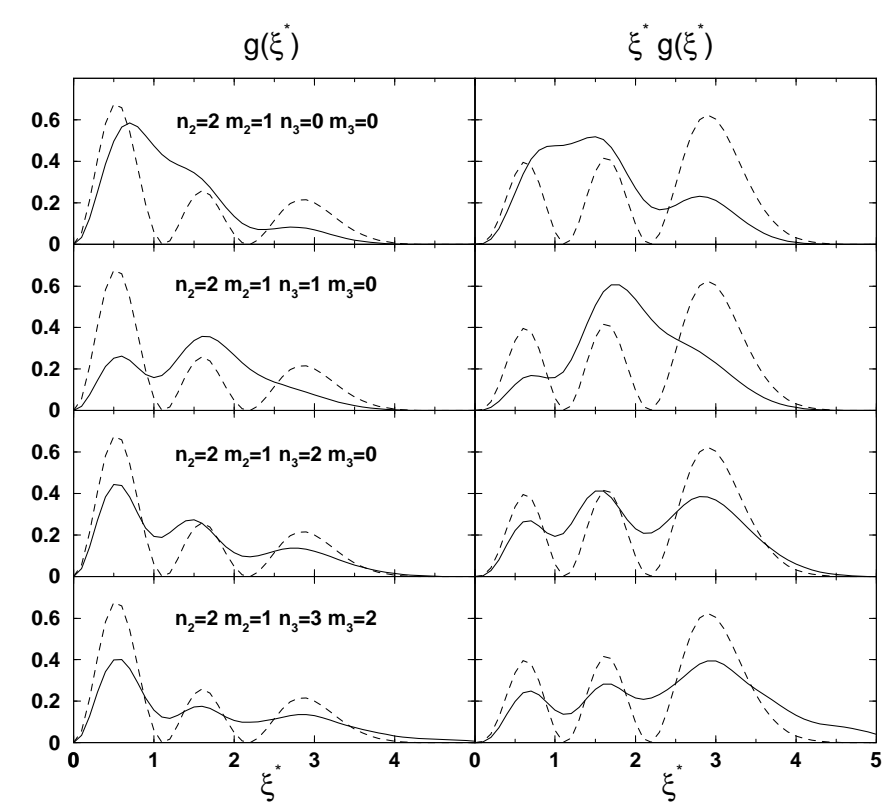

FIG. 2. The pair correlation function $g\left(\xi^{*}\right)$ versus $\xi^{*}$ for $n_{2}=2$ and different values of $n_{3}$ and $m_{3}$.

In order to get more insight into the wave function we plot in figures 1-3 the (radial) pair correlation function

$$
\begin{aligned}
G(|\xi|) & =\int_{0}^{2 \pi} d \phi_{\xi} \int d^{2} r \int d^{2} \eta\left|\Psi^{Q}\right|^{2} \\
& =\frac{\sqrt{1+\frac{4 \omega_{0}^{2}}{\omega_{B}^{2}}}}{l_{B}^{2}} g\left(\left|\xi^{*}\right|\right),
\end{aligned}
$$

where the dimensionless coordinate $\xi^{*}$ is

$$
\xi^{*}=\frac{\left(1+\frac{4 \omega_{o}^{2}}{\omega_{B}^{2}}\right)^{1 / 4}}{\sqrt{2} l_{B}} \xi .
$$

This pair correlation function gives the probability of finding two particles at a given distance in the presence of a third particle. We find that, owing to the presence of the third particle, a shell structure appears with certain maxima in the probability at special distances. The number of these maxima (shells) is defined by the quantum number $n_{2}$ and is equal to $n_{2}+1$. Therefore, the quantum number $n_{2}$ can be interpreted as the main characteristic of the number of resonant states or the number of nearest neighbors if one considers this as the onset of a liquid like behavior.

This interpretation is supported by the following reasoning. The pair correlation function starts at zero since the antisymmetrization due to Pauli-blocking induces the exchange correlation hole. A small $\xi$ expansion then yields [appendix A]

$$
\begin{aligned}
g\left(\left|\xi^{*}\right|\right) & =a\left|\xi^{*}\right|^{2 m_{2}}+\left(b+c\left|\xi^{*}\right|^{2 m_{2}}\right)\left|\xi^{*}\right|^{2}+o\left(\left|\xi^{*}\right|^{3}\right) \\
& =\left\{\begin{array}{cc}
(a+b)\left|\xi^{*}\right|^{2}, & m_{2}=1 \\
b\left|\xi^{*}\right|^{2}+a\left|\xi^{*}\right|^{2 m_{2}}, & m_{2}>1
\end{array}+o\left(\left|\xi^{*}\right|^{3}\right)\right.
\end{aligned}
$$

since $m_{2}$ is odd. The regime of $\xi^{*}<1$ can be realized, according to Eq.(23), for an arbitrary finite distance between particles by sufficiently reducing both $\omega_{0}$ and $\omega_{H}$.

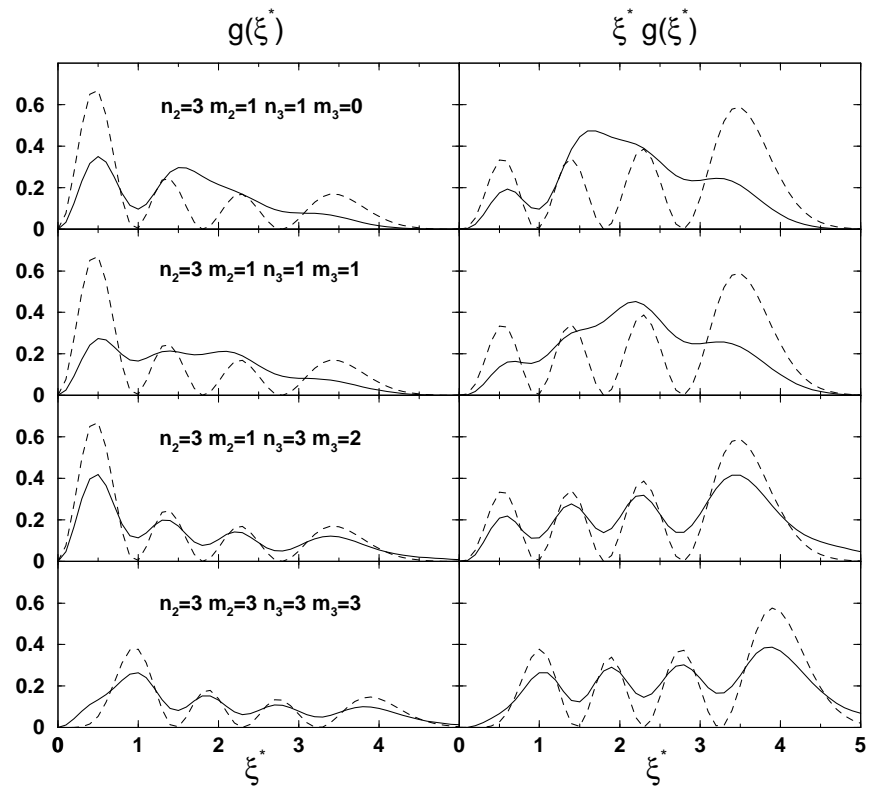

FIG. 3. The dependence of $g\left(\xi^{*}\right)$ on space coordinate for $n_{2}=3$ and different values of $n_{3}$ and $m_{3}$.

The characteristic property of the Laughlin wave function is that $g(z)$ goes to zero as $z^{2 m}$, whereas in the case of the Wigner crystal the pair-correlation function goes to zero as $1-\exp \left(-a z^{2}\right) \propto z^{2}$. This was the reason for the superiority of the variational Laughlin wave function. In our direct computation, starting from the expression of antisymmetric wave function $\Psi^{Q}$, we obtain the Wigner crystal behavior. However, the Laughlin exchange correlation hole becomes transparent if we use only the first diagonal term in $\left|\Psi^{Q}\right|^{2} \propto$ $C^{2}\left|\Psi_{n_{1}, m_{1}}\left(r ; \omega_{0}=0\right)\right|^{2}\left|\Psi_{n_{2}, m_{2}}(\xi)\right|^{2}\left|\Psi_{n_{3}, m_{3}}(\eta)\right|^{2}$ which is the first term in (A1). We obtain the analytical result

$g^{\operatorname{diag} 1}\left(\left|\xi^{*}\right|\right)=\frac{n_{2} !}{\left(n_{2}+\left|m_{2}\right|\right) !} \xi^{* 2\left|m_{2}\right|}\left(L_{n_{2}}^{\left|m_{2}\right|}\left(\xi^{* 2}\right)\right)^{2} \mathrm{e}^{-\xi^{* 2}}$.

Representing the Laguerre polynomials as $L_{n}^{m}(x)=$ $\sum_{k=0}^{n}\left(\begin{array}{c}n+m \\ n-k\end{array}\right) \frac{(-x)^{k}}{k !}$, the following asymptotic behavior of $g^{\text {diag }}$ is obtained

$$
g^{\operatorname{diag}}\left(\left|\xi^{*}\right|\right)=\frac{\left(n_{2}+\left|m_{2}\right|\right) !}{n_{2} ! m_{2} !^{2}} \xi^{* 2\left|m_{2}\right|}\left(1+o\left(\xi^{2}\right)\right) .
$$

We see that the typical $z^{2\left|m_{2}\right|}$ behavior of Laughlin states appears. Therefore, we consider the quantum number $\left|m_{2}\right|$ as the filling factor in analogy to Laughlin's exchange correlation hole behavior.

The approximation leading to this behavior has consisted in neglecting certain crossed terms. Here we should note that when considering the paircorrelation function 
we design in principle two out of the three particles differently than the third particle. In this case we don't need to antisymmetrize the wave function. One can therefore argue that all terms of Eq.(A1) except the first one do not count and the result (25) is exact.

The function $g^{\text {diag1 }}$ in Eq. (25) is plotted in Figs. 1-3 with dashed lines. We see that the places of the maxima are well described by this approximation. Note here that the approximation $g^{\text {diag1 }}$ indeed shows the Laughlin exchange correlation hole of $\xi^{2 m_{2}}$.

In order to obtain analytical results for observables we can use all three diagonal elements $\left|\Psi^{Q \text { diag }}\right|^{2}=$ $C^{2}\left|\Psi_{1}\right|^{2}\left(\left|\Psi_{2}\right|^{2}\left|\Psi_{3}\right|^{2}+\left|\tilde{\Psi}_{2}\right|^{2}\left|\tilde{\Psi}_{3}\right|^{2}+\left|\tilde{\tilde{\Psi}}_{2}\right|^{2}\left|\tilde{\tilde{\Psi}}_{3}\right|^{2}\right)$. The advantage of this approximation is that one can compute the expectation values of observables analytically and get a fairly good approximation.

We consider the mean distance of the particles

$$
\begin{aligned}
& \left\langle\frac{\left(\mathbf{r}_{1}-\mathbf{r}_{2}\right)^{2}}{2}\right\rangle=\int d^{2} \xi d^{2} r d^{2} \eta \xi^{2}\left|\Psi^{Q \text { diag }}\right|^{2} \\
& =\frac{3 C^{2} l_{B}^{2}}{\sqrt{1+\frac{4 \omega_{2}^{2}}{\omega_{B}^{2}}}}\left[\left(1+2 n_{2}+\left|m_{2}\right|\right)+\left(1+2 n_{3}+\left|m_{3}\right|\right)\right],
\end{aligned}
$$

as well as the area spanned by the three particles,

$$
\begin{aligned}
& \left\langle\frac{1}{4}\left|\left(\mathbf{r}_{2}-\mathbf{r}_{1}\right) \times\left(\mathbf{r}_{3}-\mathbf{r}_{1}\right)\right|^{2}\right\rangle=\int d^{2} \xi d^{2} r d^{2} \eta \frac{3}{4}|\xi \times \eta|^{2}\left|\Psi^{Q \text { diag }}\right|^{2} \\
& =\frac{9}{4} \frac{C^{2} l_{B}^{4}}{1+\frac{4 \omega_{o}^{2}}{\omega_{B}^{2}}}\left[\left(1+2 n_{2}+\left|m_{2}\right|\right)\left(1+2 n_{3}+\left|m_{3}\right|\right)\right] .
\end{aligned}
$$

We see that the mean distance and the mean area are determined by the quantum numbers $1+2 n_{2}+\left|m_{2}\right|$ and $1+2 n_{3}+\left|m_{3}\right|$. The mean distance is given by their sum while the mean area is given by their product. The nondiagonal terms give smaller and smaller contributions. In table I we compare the analytical approximate results

\begin{tabular}{|c|c|c|c|}
\hline$n_{2} m_{2} n_{3} m_{3}$ & $1 / C$ & dist. & area \\
\hline \multirow[t]{2}{*}{1100} & 2.24 & 2.49 & 1.13 \\
\hline & 3 & 2.5 & 1.5 \\
\hline \multirow[t]{2}{*}{1111} & 3.37 & 4.00 & 2.25 \\
\hline & 3 & 4 & 6 \\
\hline \multirow[t]{2}{*}{1121} & 1.83 & 5.00 & 7.35 \\
\hline & 3 & 5 & 9 \\
\hline \multirow[t]{2}{*}{1122} & 4.81 & 5.49 & 11.03 \\
\hline & 3 & 5.5 & 10.5 \\
\hline \multirow[t]{2}{*}{2100} & 2.79 & 3.50 & 2.11 \\
\hline & 3 & 3.5 & 2.25 \\
\hline \multirow[t]{2}{*}{2110} & 1.12 & 4.49 & 8.07 \\
\hline & 3 & 4.5 & 6.75 \\
\hline \multirow[t]{2}{*}{2111} & 1.83 & 5.00 & 7.35 \\
\hline & 3 & 5 & 9 \\
\hline \multirow[t]{2}{*}{2120} & 3.22 & 5.48 & 5.07 \\
\hline & 3 & 5.5 & 11.25 \\
\hline \multirow[t]{2}{*}{2121} & 2.67 & 5.99 & 9.94 \\
\hline & 3 & 6 & 13.5 \\
\hline \multirow[t]{2}{*}{2122} & 3.10 & 6.46 & 25.10 \\
\hline & 3 & 6.5 & 15.75 \\
\hline \multirow[t]{2}{*}{2130} & 2.17 & 6.44 & 16.12 \\
\hline & 3 & 6.5 & 15.75 \\
\hline \multirow[t]{2}{*}{2131} & 3.26 & 6.92 & 10.88 \\
\hline & 3 & 7 & 18 \\
\hline \multirow[t]{2}{*}{2132} & 3.28 & 7.26 & 28.59 \\
\hline & 3 & 7.5 & 20.25 \\
\hline \multirow[t]{2}{*}{2133} & 2.27 & 7.68 & 14.47 \\
\hline & 3 & 8 & 22.5 \\
\hline \multirow[t]{2}{*}{3110} & 1.96 & 5.48 & 9.90 \\
\hline & 3 & 5.5 & 9 \\
\hline \multirow[t]{2}{*}{3310} & 2.59 & 6.46 & 12.41 \\
\hline & 3 & 6.5 & 11.25 \\
\hline \multirow[t]{2}{*}{3111} & 1.55 & 5.99 & 14.72 \\
\hline & 3 & 6 & 12 \\
\hline \multirow[t]{2}{*}{3311} & 1.83 & 6.98 & 17.45 \\
\hline & 3 & 7 & 15 \\
\hline \multirow[t]{2}{*}{3130} & 2.58 & 7.36 & 16.19 \\
\hline & 3 & 7.5 & 21 \\
\hline \multirow[t]{2}{*}{3330} & 3.90 & 8.29 & 26.75 \\
\hline & 3 & 8.5 & 26.25 \\
\hline \multirow[t]{2}{*}{3131} & 2.20 & 7.65 & 23.02 \\
\hline & 3 & 8 & 24 \\
\hline 3331 & 2.40 & 8.46 & 23.18 \\
\hline & 3 & 9 & 30 \\
\hline 3132 & 3.92 & 8.18 & 30.50 \\
\hline & 3 & 8.5 & 27 \\
\hline 3332 & 2.28 & 8.64 & 32.36 \\
\hline & 3 & 9.5 & 33.75 \\
\hline 3133 & 2.37 & 8.15 & 23.10 \\
\hline & 3 & 9 & 30 \\
\hline 3333 & 3.70 & 9.29 & 41.88 \\
\hline & 3 & 10 & 37.5 \\
\hline
\end{tabular}
(27) and (28) with the numerical value of the full wave function. The agreement especially for the mean distance suggests that this is quite a good approximation.
TABLE I. The normalization constant $C$, the dimensionless mean distance and the area for different quantum numbers. The corresponding second lines give the analytical values (27) and (28) respectively. 
Although the states in high magnetic fields are characterized by spin alignment and are therefore described by the quartet states, we now give for completeness the other possible state, the doublet state. The wave functions corresponding to the doublet states can be constructed according to Young's scheme [18]. For the state $\left|S=1 / 2, s_{z}=1 / 2\right\rangle$ it is given in Fig. 4. The expressions corresponding to the configurations (a), (b), and (c) in Fig. 4 are obtained by antisymmetrizing the spin wave function with respect to the row indices and symmetrizing with respect to the column ones,

$$
|1 / 2,1 / 2\rangle_{a}=c\left(\alpha_{2}\left\{\alpha_{1} \beta_{3}-\alpha_{3} \beta_{1}\right\}+\alpha_{1}\left\{\alpha_{2} \beta_{3}-\alpha_{3} \beta_{2}\right\}\right)
$$

and correspondingly for (b) and (c). It is possible to see that one particular spin wave function transforms into the other one when two particle indices are interchanged, forming a group.

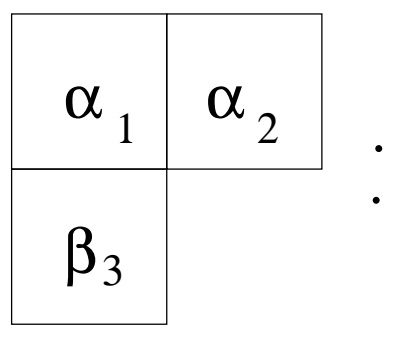

a)

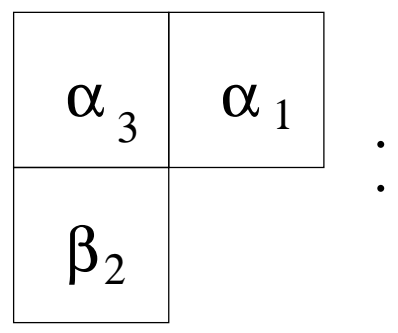

b)
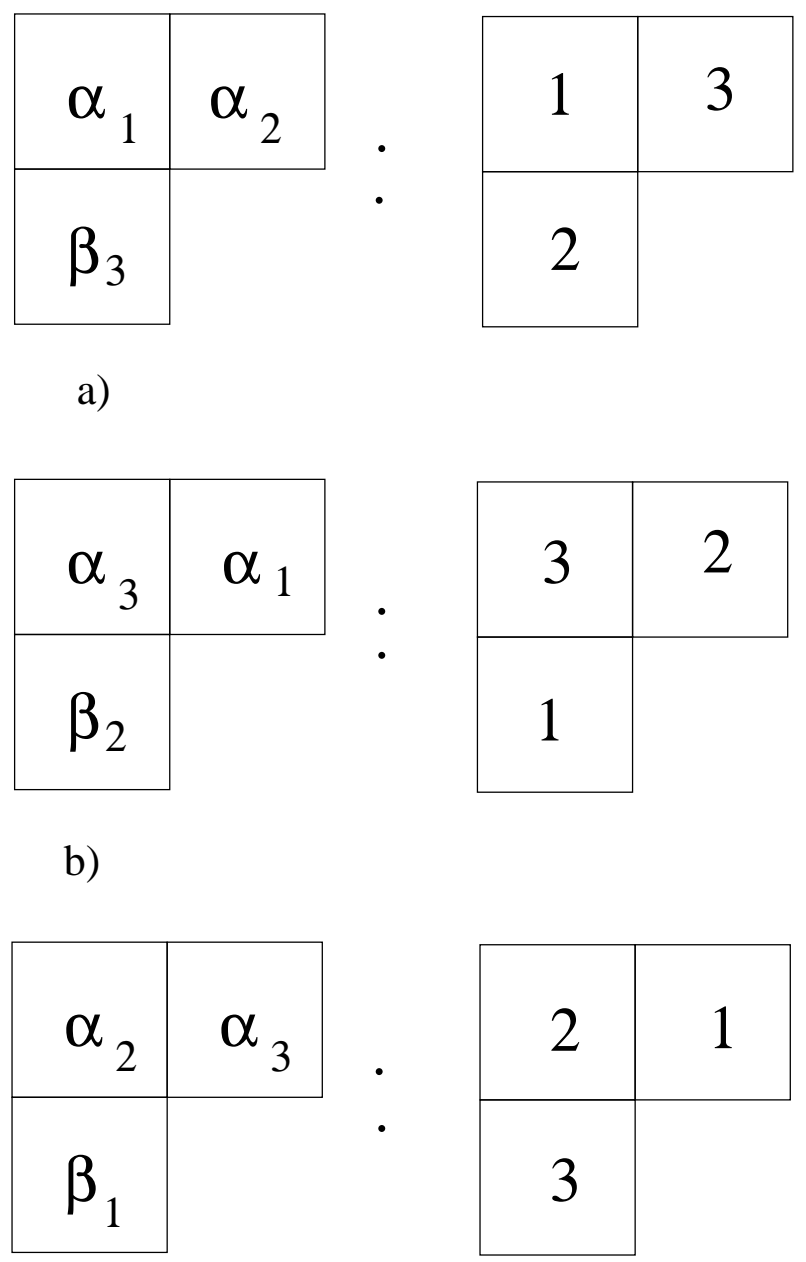

c)
FIG. 4. The particular Young diagrams contributing to the spin state $\left|S=1 / 2, s_{z}=1 / 2\right\rangle$ are shown on the left with the corresponding contributions to the spatial wave function on the right. The indices in one row are understood to be symmetrized and the indices in one column are antisymmetrized. The diagrams for $\left|S=1 / 2, s_{z}=-1 / 2\right\rangle$ are obtained by interchanging $\alpha \leftrightarrow \beta$.

The spatial wave functions for each spin diagram are obtained by transposition of the Young tableaux of Fig. 4 . Once again, the elements in a row must be symmetrized and the elements in a column antisymmetrized. The spatial wave function corresponding to (a) in Fig. 4, Eq. (29), reads

$$
\begin{aligned}
& \Psi_{a}^{D}\left(\mathbf{r}_{1}, \mathbf{r}_{2}, \mathbf{r}_{3}\right)=C \Psi_{n_{1}, m_{1}}\left(r ; \omega_{0}=0\right) \\
& \times\left\{\Psi_{n_{2}, m_{2}}(-\xi) \Psi_{n_{3}, m_{3}}(\eta)+\Psi_{n_{2}, m_{2}}(\tilde{\xi}) \Psi_{n_{3}, m_{3}}(\tilde{\eta})\right. \\
& \left.-\Psi_{n_{2}, m_{2}}(\xi) \Psi_{n_{3}, m_{3}}(\eta)-\Psi_{n_{2}, m_{2}}(-\tilde{\tilde{\xi}}) \Psi_{n_{3}, m_{3}}(\tilde{\tilde{\eta}})\right\}
\end{aligned}
$$

Denoting only the spatial indices, the coordinates $\xi, \eta$ defined in Eq. (2) can be abbreviated as $(1,23)$. The coordinate $\tilde{\xi}, \tilde{\eta}$ of $(20)$ would correspond to $(2,31), \tilde{\tilde{\xi}}, \tilde{\tilde{\eta}}$ to $(3,12)$, and $-\tilde{\xi}, \tilde{\eta}$ to $(3,21)$ respectively. In this short notation the three orbital wave functions of figure (4) read

$$
\begin{aligned}
& \Psi_{a}^{D}=(2,13)+(2,31)-(1,23)-(1,32) \\
& \Psi_{b}^{D}=(1,32)+(1,23)-(3,21)-(3,12) \\
& \Psi_{c}^{D}=(3,21)+(3,12)-(2,31)-(2,13) .
\end{aligned}
$$

Multiplying the spin and spatial components and adding all three particular expressions, we get the final wave function for the doublet state. The final result depends on the parity of $m_{2}$. For $m_{2}$ odd it reads

$$
\begin{aligned}
& \Psi^{D}\left(\mathbf{r}_{1}, \mathbf{r}_{2}, \mathbf{r}_{3}\right)=C_{1} \Psi_{n_{1}, m_{1}}\left(r ; \omega_{0}=0\right) \\
& \times\left\{\left(\beta_{1} \alpha_{2} \alpha_{3}+\alpha_{1} \beta_{2} \alpha_{3}-2 \alpha_{1} \alpha_{2} \beta_{3}\right) \Psi_{n_{2}, m_{2}}(\xi) \Psi_{n_{3}, m_{3}}(\eta)\right. \\
& +\left(\alpha_{1} \beta_{2} \alpha_{3}+\alpha_{1} \alpha_{2} \beta_{3}-2 \beta_{1} \alpha_{2} \alpha_{3}\right) \Psi_{n_{2}, m_{2}}(\tilde{\xi}) \Psi_{n_{3}, m_{3}}(\tilde{\eta}) \\
& \left.+\left(\beta_{1} \alpha_{2} \alpha_{3}+\alpha_{1} \alpha_{2} \beta_{3}-2 \alpha_{1} \beta_{2} \alpha_{3}\right) \Psi_{n_{2}, m_{2}}(\tilde{\tilde{\xi}}) \Psi_{n_{3}, m_{3}}(\tilde{\tilde{\eta}})\right\}
\end{aligned}
$$

while for $m_{2}$ even the wave function is given by

$$
\begin{aligned}
& \Psi^{D}\left(\mathbf{r}_{\mathbf{1}}, \mathbf{r}_{\mathbf{2}}, \mathbf{r}_{3}\right)=C_{2} \Psi_{n_{1}, m_{1}}\left(r ; \omega_{0}=0\right) \\
& \times\left\{\left(\alpha_{1} \beta_{2} \alpha_{3}-\beta_{1} \alpha_{2} \alpha_{3}\right) \Psi_{n_{2}, m_{2}}(\xi)\right) \Psi_{n_{3}, m_{3}}(\eta) \\
& +\left(\alpha_{1} \alpha_{2} \beta_{3}-\alpha_{1} \beta_{2} \alpha_{3}\right) \Psi_{n_{2}, m_{2}}(\tilde{\xi}) \Psi_{n_{3}, m_{3}}(\tilde{\eta}) \\
& \left.+\left(\beta_{1} \alpha_{2} \alpha_{3}-\alpha_{1} \alpha_{2} \beta_{3}\right) \Psi_{n_{2}, m_{2}}(\tilde{\tilde{\xi}}) \Psi_{n_{3}, m_{3}}(\tilde{\tilde{\eta}})\right\} .
\end{aligned}
$$

It is easy to check that the expression for the probability distribution $\left|\Psi^{D}\right|^{2}$ in the doublet state differs from the one for the quartet state by the coefficients in front of the crossed terms. Our estimate shows that the character of quantization of the two-particle correlator, the 
cluster area, mean distance for doublet states is qualitatively similar to that for quartet states. Since the contributions of the crossed terms in the quartet states are smaller than those in the doublet states, the maxima in the pair correlation function are more pronounced in the former case.

\section{CONCLUSIONS}

The investigation of the three-particle problem in the presence of magnetic fields is essential to understand the phenomenon of the fractional quantum Hall effect [2]. In this paper we have solved exactly the problem of three identical particles interacting via a spring potential in $2 \mathrm{D}$ space in the presence of an external magnetic field,neglecting the Coulomb interactions. We have shown that the wave function acquires the Jastrow prefactor even in the case of an attractive potential as a consequence of the anti-symmetric character of the total wave function. Calculations of the pair correlation function, the area of the three-particle cluster and the mean distance show a quantization of these parameters. Particularly, the pair correlation function exhibits maxima and minima controlled by a quantum number.

We have also found that the exchange correlation hole at small distances shows the behavior known from the Wigner lattices rather than the Laughlin behavior if we start from the antisymmetrized wave function. However, neglecting certain terms based on the physical consideration that the antisymmetrization of the wave function fails when two particles are fixed for the pair correlation, we obtain exactly the Laughlin exchange correlation hole. The corresponding filling factor is identified with a quantum number appearing in the exact solution of the model solved here.

\section{APPENDIX A: SMALL DISTANCE EXPANSION OF THE PAIRCORRELATION FUNCTION}

The expansion of the paircorrelation function (22) for small $\xi$ can be performed straightforwardly with the help of MATHEMATICA. After trivial integrations about $d^{2} r$ we have to calculate

$$
\begin{aligned}
g\left(\left|\xi^{*}\right|\right)= & \int d^{2} \eta^{*}\left(\left|\Psi_{2}\right|^{2}\left|\Psi_{3}\right|^{2}+2\left|\tilde{\Psi}_{2}\right|^{2}\left|\tilde{\Psi}_{3}\right|^{2}\right. \\
& \left.+2 \operatorname{Re}\left\{2 \Psi_{2} \Psi_{3} \tilde{\Psi}_{2}^{*} \tilde{\Psi}_{3}^{*}+2 \tilde{\Psi}_{2} \tilde{\Psi}_{3} \tilde{\tilde{\Psi}}_{2}^{*} \tilde{\widetilde{\Psi}}_{3}^{*}\right\}\right)
\end{aligned}
$$

where we have used symmetries in the variables and abbreviated $\tilde{\Psi}_{2}=\Psi_{n_{2}, m_{2}}(\tilde{\xi}), \tilde{\tilde{\Psi}}_{3}=\Psi_{n_{3}, m_{3}}(\tilde{\tilde{\eta}})$ and similar for the other combinations. Therefore we express the occurring variables (20) in the wave function in terms of the integration variables

$$
\begin{aligned}
|\tilde{\xi}|^{2} & =\frac{1}{4}\left[3|\eta|^{2}+|\xi|^{2}-2 \sqrt{3}|\eta||\xi| \cos \left(\phi_{\xi}-\phi_{\eta}\right)\right] \\
\mathrm{e}^{i \phi_{\tilde{\xi}}} & =\frac{1}{2|\tilde{\xi}|}\left(\sqrt{3}|\eta| \mathrm{e}^{i \phi_{\eta}}-|\xi| \mathrm{e}^{i \phi_{\xi}}\right)
\end{aligned}
$$

and similarly for $\tilde{\eta}$ as well as $\tilde{\tilde{\eta}}$ and $\tilde{\tilde{\xi}}$. Using the dimensionless coordinates (23) in the wave function (14) and changing variables (A2), the integral (A1) is quite massy. However, expanding in small $\xi^{*}$ the angular dependence $\phi_{\eta}-\phi_{\xi}$ drops out and we obtain the form (24)

$$
g\left(\left|\xi^{*}\right|\right)=a\left|\xi^{*}\right|^{2 m_{2}}+\left(b+c\left|\xi^{*}\right|^{2 m_{2}}\right)\left|\xi^{*}\right|^{2}+o\left(\left|\xi^{*}\right|^{3}\right)
$$

where the remaining modulus integral $d|\eta|$ can be performed for the constants $a$ and $c$ with the result

$$
\begin{aligned}
& a=2 \frac{\left(n_{2}+m_{2}\right) !}{m_{2} !^{2} n_{2} !} \\
& c=-\frac{2\left(n_{2}+m_{2}\right) !}{n_{2} ! m_{2} !}\left(2 \frac{n_{2}}{\left(1+m_{2}\right) !}+\frac{1}{m_{2} !}\right) .
\end{aligned}
$$

Therefore we see that the constant $a$ is not vanishing which means that the Laughlin liquid behavior is not resumed if full antisymmetrization is pertained for the pair correlation function.

For completeness let us also give the constant $b$ which integral can be done only numerically $\left[x=\left|\eta^{*}\right|^{2}\right]$

$$
\begin{aligned}
b= & \frac{(-1)^{2 m_{3}} 3^{m_{2}-1}}{4^{m_{2}+m_{3}}} \frac{n_{2} ! n_{3} !}{\left(m_{2}+n_{2}\right) !\left(m_{3}+n_{3}\right) !} \mathcal{I} \\
\mathcal{I}= & \int_{0}^{\infty} d x x^{m_{2}+m_{3}-1} \mathrm{e}^{-x} \\
& \times\left\{8\left(m_{2}-3 m_{3}\right)^{2} L_{n_{2}}^{m_{2}}\left(\frac{3 x}{4}\right)^{2} L_{n_{3}}^{m_{3}}\left(\frac{x}{4}\right)^{2}\right. \\
& +12\left(m_{2}-3 m_{3}\right) x L_{n_{2}}^{m_{2}}\left(\frac{3 x}{4}\right) L_{n_{3}}^{m_{3}}\left(\frac{x}{4}\right) \\
& \times\left(L_{n_{2}}^{m_{2}}\left(\frac{3 x}{4}\right) L_{n_{3}-1}^{m_{3}+1}\left(\frac{x}{4}\right)-L_{n_{2}-1}^{m_{2}+1}\left(\frac{3 x}{4}\right) L_{n_{3}}^{m_{3}}\left(\frac{x}{4}\right)\right) \\
& \left.+9 x^{2}\left(L_{n_{2}}^{m_{2}}\left(\frac{3 x}{4}\right) L_{n_{3}-1}^{m_{3}+1}\left(\frac{x}{4}\right)-L_{n_{2}-1}^{m_{2}+1}\left(\frac{3 x}{4}\right) L_{n_{3}}^{m_{3}}\left(\frac{x}{4}\right)\right)^{2}\right\}
\end{aligned}
$$

[1] R. B. Laughlin, Phys. Rev. Lett. 50, 1395 (1983).

[2] R. B. Laughlin, in The Quantum Hall Effect, edited by R. E. Prange and S. M. Girvin (Springer, Berlin, 1987).

[3] F. Calogero, J. Math. Phys. 10, 2197 (1969).

[4] F. D. M. Haldane, in Correlation effects in low dimensional systems, edited by A. Okiji and N. Kawakami (Springer, Berlin, 1994). 
[5] F. Calogero and C. Marchioro, J. Math. Phys. 14, 182 (1973).

[6] A. Khare and K. Ray, Phys. Lett. A 230, 139 (1997).

[7] A. Khare, Phys. Lett. A 245, 14 (1998).

[8] B. D. Simons, P. A. Lee, and B. L. Altshuler, Phys. Rev. Lett. 72, 64 (1994).

[9] B. L. Altshuler, P. A. Lee, and R. A. Webb, Mesoscopic phenomena in Solids (North-Holland, Amsterdam, 1991).

[10] E. P. Nakhmedov and K. Morawetz, Phys. Rev. B 66, 195333 (2001).

[11] L. D. Faddeev, in Israel Program for Scientific Translation (PUBLISHER, Jerusalem, 1965).

[12] L. R. Dodd, J. Math. Phys. 11, 207 (1970).

[13] J. E. van Himbergen and J. A. Tjon, Physica 76, 503 (1974).

[14] C. K. Majumdar and I. Bose, J. Math. Phys. 19, 2187 (1978).

[15] J. L. Friar, B. F. Gibson, and G. L. Payne, Phys. Rev. C 22, 284 (1980).

[16] N. Barnea and V. B. Mandelzweig, Phys. Rev. C 45, 1458 (1992).

[17] N. Barnea and V. Mandelzweig, Phys. Rev. C 49, 2910 (1994).

[18] L. Landau and E. M. Lifschitz, Quantum Mechanics: Non-Relativistic Theory (Vol. III, Pergamon, Oxford, 1977). 